\title{
Acute Pulmonary Embolism Masquerading as Acute Inferior Myocardial Infarction
}

\author{
Chukwudi Obiagwu $^{\mathrm{a}}$, Jinu John ${ }^{\mathrm{b}, \mathrm{c}}$, Lou Mastrine ${ }^{\mathrm{b}}$, Elliot Borgen ${ }^{\mathrm{b}}$, Jacob Shani ${ }^{\mathrm{b}}$
}

\begin{abstract}
It is always important to maintain a high index of suspicion in the diagnosis of acute pulmonary embolism (PE). Death in patients with undiagnosed PE was shown to be $4-6$ times higher than in those with a definite diagnosis and treatment. The ECG as much as it has great utility, unfortunately lacks sensitivity and specificity, providing findings that might serve as red herrings to the unsuspecting physician by suggesting an alternative diagnosis. This report describes a woman whose EKG showed ST segment elevations suggestive of acute inferior wall myocardial infarction (MI), but eventually was found to have a sub-massive PE.
\end{abstract}

Keywords: Pulmonary embolism; Myocardial infarction; Echocardiogram in pulmonary embolism

\section{Introduction}

It is of utmost importance to diagnose acute pulmonary embolism (PE) early and commence treatment. The ECG is still simple, easy to use and useful in the diagnosis of acute PE, although it should be noted that findings have low sensitivity and specificity. Echocardiography can be very helpful in differentiating massive acute PE from acute myocardial infarction (MI), especially in resource-constrained regions. Physicians should always remember to assess for acute PE

Manuscript accepted for publication October 22, 2013

${ }^{a}$ Department of Medicine, Maimonides Medical Center, Brooklyn, NY 11219, USA

${ }^{\mathrm{b}}$ Department of Cardiology, Maimonides Medical Center, Brooklyn, NY 11219, USA

${ }^{\mathrm{c}}$ Corresponding author: Jinu John, Department of Cardiology, 4802

Tenth Avenue, Brooklyn, NY 11219, USA.

Email: doctorjacob@gmail.com

doi: http://dx.doi.org/10.14740/jmc1584e even while suspecting acute MI.

\section{Case Report}

An 85-year-old woman with a history of diabetes mellitus type II, hypertension and hyperlipidemia was brought to the emergency department (ED) with acute onset shortness of breath (SOB) and mid sternal chest pain of $1 \mathrm{~h}$ duration. She had been complaining of intermittent chest pain and SOB over the previous 1 week. She described the pain as pressure like and non-radiating. She denied nausea, vomiting, leg swelling, cough or fever. On admission to the ED, her heart rate was $87 / \mathrm{min}$, respiratory rate was $16 / \mathrm{min}$, oxygen saturation was $94 \%$ on room air, blood pressure was $140 / 90$ $\mathrm{mmHg}$, body temperature was $98.1 \mathrm{~F}$ and Glasgow coma scale was E4V5M6. Cardiac and respiratory exams were unremarkable. Her home medications included aspirin, enalapril and metoprolol.

A 12-lead EKG performed en route to the hospital revealed sinus rhythm and ST segment elevations in inferior leads, an S1Q3T3 (Fig. 1). Cardiac troponin and CKMB were elevated, $2.24 \mathrm{ng} / \mathrm{mL}$ and $6.6 \mathrm{ng} / \mathrm{mL}$ respectively. The chest X-ray showed increased prominence of the hilar regions. The patient was heparinized and aspirin was administered. As the patient continued to have sub-sternal chest pain, she was taken to the cardiac catheterization laboratory for emergent coronary angiography, which showed an ulcerated plaque in the proximal right coronary artery (pRCA) with a $60 \%$ stenosis (Fig. 2). A possibility that she might have had a thrombus in the region of the ulcerated plaque which autolyzed prior to angiography was considered and a drug eluting stent was placed in the pRCA with a good result. Subsequently the patient was transferred to the cardiac ICU.

But chest pain and SOB persisted post procedure, and her oxygen saturation continued to be low. An arterial blood gas (ABG) revealed a huge alveolar arterial (A-a) gradient. A bedside transthoracic echocardiogram (TTE) was carried out which revealed normal LV ejection fraction, severely reduced RV systolic function, moderately enlarged RV and right atrium, and moderately elevated pulmonary artery systolic pressure (Fig. 3). This was highly suspicious for PE. A 


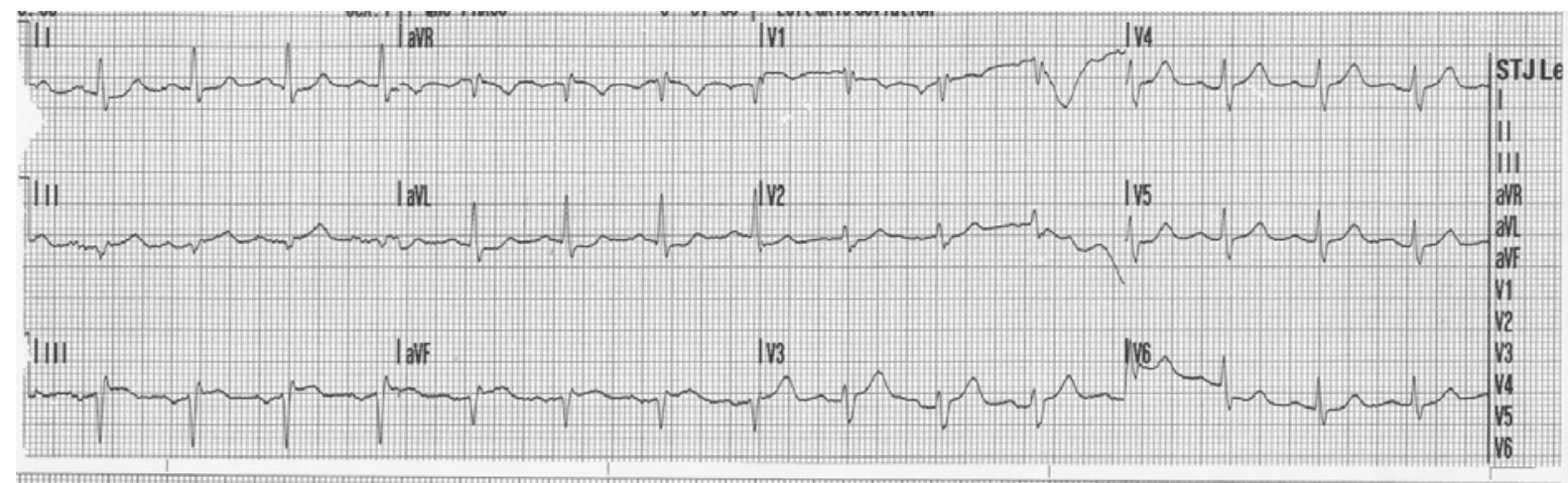

Figure 1. 12-lead EKG done en route to the hospital showing ST elevations and q waves in inferior leads.

CT angiogram of the chest showed extensive bilateral PE, including a saddle pulmonary embolus (Fig. 4). Duplex ultrasound scan of the lower extremities showed acute partial thrombosis in the proximal segment of right popliteal vein. Although a surgical embolectomy was offered, as the patient declined, it could not be performed. An inferior vena cava filter was placed to prevent further embolism. She received anticoagulant therapy with heparin. The rest of her hospital stay was uneventful. She was discharged 10 days later in a fair condition.

\section{Discussion}

The clinical presentation of acute PE is often misleading and confusing, thus leading to misdiagnosis. Surface ECG readings are altered in about $70 \%$ of cases of PE [1]. Numerous ECG findings have been reported, with sinus tachycardia being the most common [2]. Findings such as the S1Q3T3 pattern lack sensitivity and specificity, and also show no correlation with the severity of PE $[2,3]$. Several studies have stated that T-wave inversion in lead III, aVF and precordial leads is most often associated with massive PE and/or PE with RV dysfunction, ascribing a high sensitivity, specificity, positive and negative predictive value to these findings [3-6]. Negative $T$ waves in both leads III and $V_{1}$ have been reported to accurately differentiate acute PE from acute coronary syndrome [4].

Echocardiographic criteria potentially useful for diagnosing acute PE are based either on disturbed RV ejection pattern (the 60-60 sign: pulmonary artery acceleration time of $<60 \mathrm{~ms}$ and tricuspid valve peak systolic gradient $<60$ $\mathrm{mmHg}$ ) or on depressed contractility of the RV free wall compared with its apex (the McConnell sign) and they seem to have a higher PPV despite pre-existing cardiorespiratory diseases. Thus, a normal TTE makes PE with significant he-
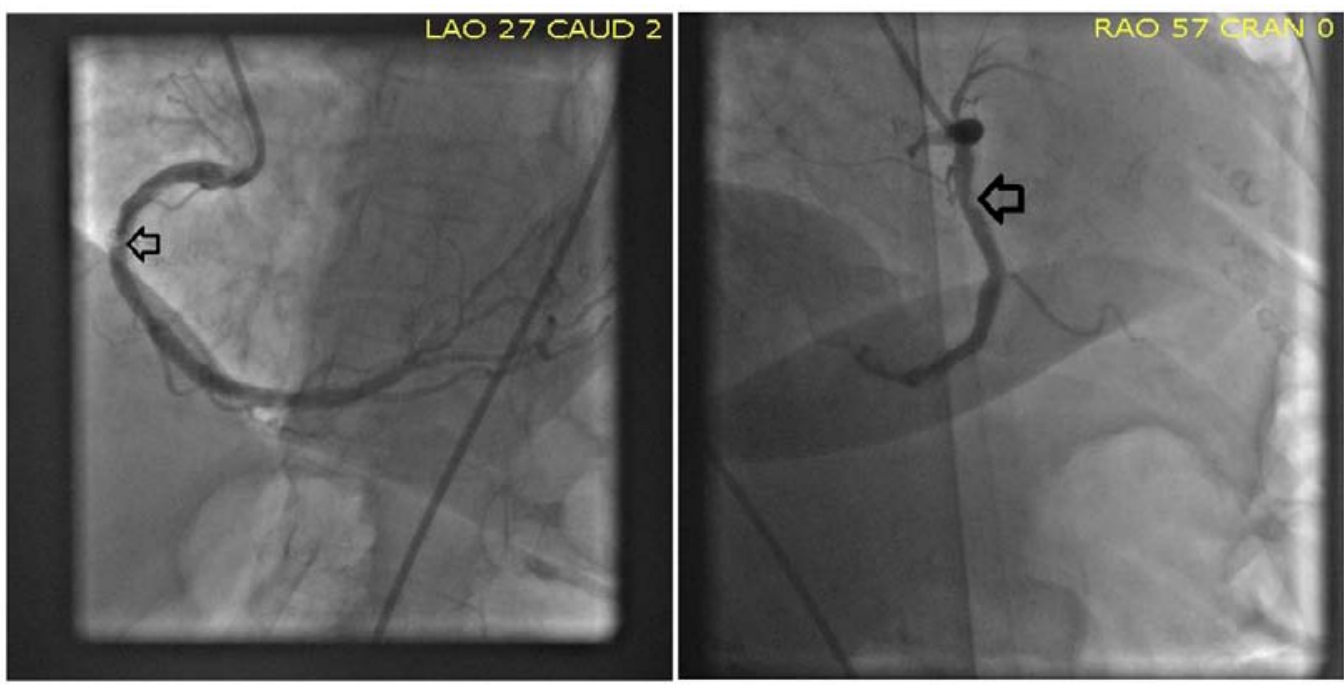

Figure 2. Coronary angiogram showing a possible ulcerated plaque in the right coronary artery. 


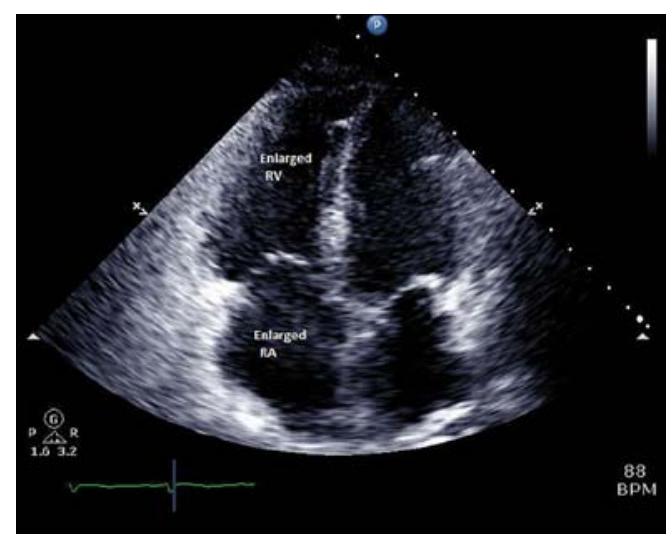

Figure 3. Echocardiogram (apical four chamber view) showing dilated right ventricle and right atrium.

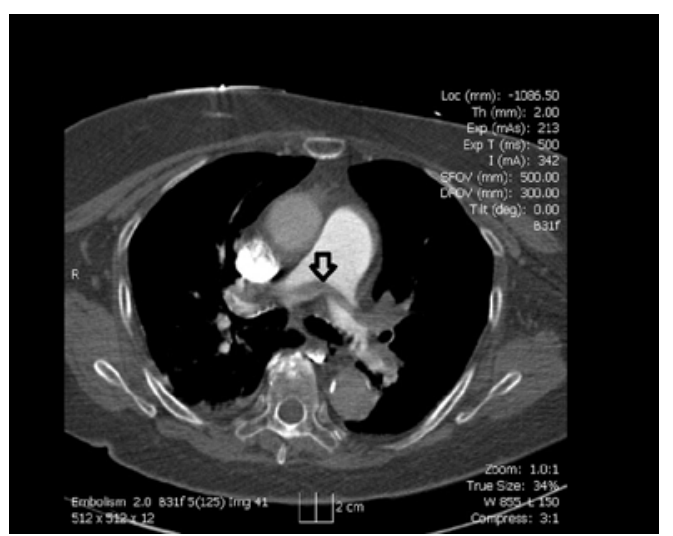

Figure 4. CT angiogram showing saddle embolus in the main pulmonary artery. modynamic compromise very unlikely [6].

We think that the ulcerated plaque on coronary angiography was an incidental finding and not the primary cause of her symptoms. Retrospectively thinking, if our patient had a bedside echocardiogram or an ABG on admission in the ED, possibly PE might have been diagnosed earlier. Although our patient had a good outcome eventually, that should have been the ideal sequence of events. Also our patient had no tachycardia on presentation possibly due to metoprolol use. There was no leg pain even though we later found her to have DVT. The maximum troponin was only 3.5 (which can happen in $\mathrm{PE}$ also due to RV strain) and the subsequent EKGs showed no ischemic changes, again proving that she did not have an acute coronary syndrome.

Our aim is to draw the attention of clinicians to APE that mimics AMI, in this case, an elderly woman with an atypical presentation. Our initial treatment with heparin seemed to improve her clinical condition; however, placing her on antiplatelet alone post coronary angiogram led to reemergence of her symptoms.

\section{Conflict of Interest}

None.

\section{References}

1. Kosuge M, Kimura K, Ishikawa T, Ebina T, Hibi K, Kusama I, Nakachi T, et al. Electrocardiographic differentiation between acute pulmonary embolism and acute coronary syndromes on the basis of negative $\mathrm{T}$ waves. Am J Cardiol. 2007;99(6):817-821.

2. Laack TA, Goyal DG. Pulmonary embolism: an unsuspected killer. Emerg Med Clin North Am. 2004;22(4):961-983.

3. Cheng AS, Money-Kyrle A. Instructive ECG series in massive bilateral pulmonary embolism. Heart. 2005;91(7):860-862.

4. Punukollu G, Gowda RM, Vasavada BC, Khan IA. Role of electrocardiography in identifying right ventricular dysfunction in acute pulmonary embolism. Am J Cardiol. 2005;96(3):450-452.

5. Sarin S, Elmi F, Nassef L. Inverted T waves on electrocardiogram: myocardial ischemia versus pulmonary embolism. J Electrocardiol. 2005;38(4):361-363.

6. Guidelines on diagnosis and management of acute pulmonary embolism. Task Force on Pulmonary Embolism, European Society of Cardiology. Eur Heart J. 2000;21(16):1301-1336. 